\title{
Stability of manganese dioxide by guar gum in the absence or presence of surfactants
}

\author{
E. Grządka
}

Received: 14 May 2013/Accepted: 22 January 2014/Published online: 31 January 2014

(C) The Author(s) 2014. This article is published with open access at Springerlink.com

\begin{abstract}
Stability of the manganese dioxide $\left(\mathrm{MnO}_{2}\right)$ suspensions by non-ionic guar gum (GG) in the absence or presence of the surfactants: anionic sodium dodecyl sulphate (SDS), cationic hexadecyltrimethylammonium bromide (CTAB) and non-ionic Triton $\mathrm{X}-100$ (t-octylphenoxypolyethoxyethanol) and their equimolar mixtures (SDS/TX-100; CTAB/TX-100) was measured using turbidity. The obtained results of the manganese dioxide suspensions stability were discussed together with the adsorption data and with the data concerning the thicknesses of the adsorption layers. In order to gain more information about the structure of the electric double layer surface charge density and the zeta potential measurements were performed. The obtained results show that the addition of guar gum to the $\mathrm{MnO}_{2}$ suspensions increases $\mathrm{MnO}_{2}$ stability. The larger this increase is, the higher is the concentration of the polymer (concentration range 10-200 ppm). Moreover, the addition of single surfactants also causes the increase in the effectiveness of stabilizing the manganese dioxide suspensions. The reason for that is formation of multilayer complexes between the polymer and the surfactants. In such a system both the adsorption of polymer and the
\end{abstract}

E. Grządka ( $₫)$

Department of Chemistry, Faculty of Radiochemistry and Chemistry of Colloids, Maria Curie-Skłodowska

University, M. Skłodowskiej-Curie 3 Sq., 20-031 Lublin,

Poland

e-mail: egrzadka@wp.pl thickness of polymer adsorption layer increase. The greatest increase in the stability of $\mathrm{MnO}_{2} / \mathrm{GG}$ suspensions was provided by the mixture of anionic and nonionic surfactants due to a strong synergistic effect. Also, mixing the polymer and two surfactants reduces the stability of the suspension.

Keywords Stability of colloids - Guar gum SDS CTAB TX-100 $\cdot$ Manganese dioxide

\section{Introduction}

Colloidal dispersions find many interesting applications in different branches of industry (Napper 1983). However, the problem of such systems is their stability. The theory proposed by Derjaguin, Landau (1941), Verwey and Overbeek (1948) (DLVO theory) considers the stability of colloidal dispersion using the net interaction energy $(V)$ given by the algebraic sum of negative contribution due to the London-Van der Waals attraction $\left(V_{A}\right)$ and a positive one due to the electrostatic repulsion $\left(V_{R}\right) ;\left(V=V_{A}+V_{R}\right)$. Because $V_{R}$ is very sensitive to the electrolyte concentration ( $V_{A}$ is not), the stability of colloidal dispersion can be controlled by the ionic strength of background electrolyte added to the dispersion. The electrolyte has the ability to screen repulsive forces. Aqueous dispersion of solid particles can be electrostatically stabilized even without a polymer. Such a situation occurs if the range of the electrostatic repulsion is higher than that 
of the attractive Van der Waals forces. The colloidal dispersions in nonpolar solvents are usually unstable in the absence of polymers. In polar and apolar solvents the colloidal dispersions can be sterically stabilized by polymers which adsorb on the surface of the solids. However, natural or synthetic high molecular weight substances, because of their high interfacial activity, can stabilize or flocculate the colloidal suspensions. Increase or decrease of stability depends on the molecular weight of polymer and on its concentration (Wiśniewska et al. 2013). In some applications flocculation is desired, for example in purification of water suspensions and papermaking industry or mineral processing, but in majority of cases polymers are added to the colloidal dispersion to induce the increase of stability. Stable colloids are needed in ceramics, food, cosmetics, drugs, paints and varnishes (Napper 1983). Colloid stability is observed when the polymer layer created on the surface of a solid is thinner than the electric double layer and then the adsorbed polymer adopts a tethered brush structure (Ouali et al. 1999). Aggregation of particles of a solid covered by a polymer is induced by formation of interparticle links especially when the adsorbed polymer creates a loopy structure (Ouali et al. 1999).

In most cases polymers are added to the colloidal dispersion in order to induce stability or flocculation but recently mixtures of polymers and surfactants have been used more widely (Somasundaran et al. 1998; Fan et al. 1999; Terayama et al. 2001; Besra et al. 2002; Şakar-Deliormanl1 2007). The first reason for that is higher effectiveness of the polymer-surfactant mixtures especially in stabilization of drug particles. The second is the fact that the desired polymer conformation can be influenced by the addition of the second polymer or surfactant as well as changing $\mathrm{pH}$, ionic strength or type of electrolyte. It is generally known that the adsorption in the systems where not only a polymer but also a surfactant is present depends on the chemical character of these two adsorbates as well as on the properties of the solid surface (Grzadka 2011). In the systems where a polymer and the surface active agents are present three adsorption cases can be distinguished: (1) competitive adsorption: both the polymer and surfactant have the affinity for the surface but not for each other (2) complex formation: only one of these two substances has the affinity for the surface but they can bind each other (3) competitive adsorption together with complex formation: both the polymer and the surfactant have the affinity for the surface and to each other (Grzadka 2011). The stability of the colloidal suspensions may be estimated by many different methods as for example sedimentation rate, electron microscopy, photon correlation spectrometry, particle counting, electron spin resonance, turbidity, spectrophotometry, potentiometric titration and zeta potential measurements (Somasundaran et al. 1998; Wiśniewska et al. 2010, 2012a, b, 2013). Somasundaran et al. (1998) studied the role of conformation and orientation of surfactants and polymers in controlling flocculation and dispersion of aqueous and non-aqueous (toluene) $\mathrm{Al}_{2} \mathrm{O}_{3}$ suspensions. They found that in the aqueous systems the surfactant adsorption generates hydrophobic patches and imparts sufficient hydrophobicity to the particles, but in organic media, where the surface aggregation is not observed, higher surface coverages are required to obtain sufficient hydrophobicity. Moreover, in nonpolar systems the stability can also result from the presence of trace polar impurities, such as water, that can induce ionization in the adsorbed layer. Fan et al. (1999) studied competitive adsorption of anionic surfactant (SDS) and anionic polymer (polyacrylic acid) on alumina particles as well as the stability of these suspensions with the particular attention to the conformation of the polymer. They found that the sequence of addition is of crucial importance in determining the dispersion/flocculation of this system. When SDS was added first, both PAA conformation and suspension stability varied with the SDS concentration. Whereas PAA was added first, SDS macromolecules had no effect on the polymer conformation and suspension stability. Terayama et al. (2001) found that the mixture of SDS and cellulose provides more stable aqueous dispersion of CT112 (5-(3-ethoxy-4pentyloxyphenyl)-2,4-thiazolid-inedione) than SDS or cellulose alone. These results were obtained using a turbiscan MA2000. According to these scientists, the steric hindrance was caused by the adsorption of polymer as well as the electrostatic repulsion was caused by the adsorption of SDS. Besra et al. (2002) studied flocculation and dewatering of kaolin suspensions by anionic polyacrylamide in the presence of surfactants (SDS, CTAB, TX-100). They stated that the simultaneous addition of PAM either with SDS or Triton X-100 increases the settling rate, whereas when added from its mixture with CTAB it decreases. The reason is the electrostatic interaction and binding of 
CTAB to PAM-A leading to complex formation, which reduces its flocculating properties. Esumi et al. (2000) studied flocculation of alumina in the presence of the mixture of anionic and nonionic surfactants. They noticed that for the dual systems enhancement of the adsorption of one surfactant would occur by the adsorption of another one. Şakar-Deliormanlı (2007) investigated the effect of cationic surfactant (dodecylamine) and nonionic triblock copolymer (PEOPPO-PEO) on the dispersion and flocculation behaviour of aqueous silica suspensions and stated that these processes depend on the type and concentration of surfactant surfactant/polymer ratio and surfactant addition sequence to the system.

The aim of this work was to investigate the influence of guar gum and surfactants (anionic SDS, cationic CTAB and nonionic Triton X-100) as well as the mixtures of surfactants (SDS/Triton X-100; CTAB/Triton X-100) on the stability of the $\mathrm{MnO}_{2}$ suspensions using turbidimetry. The results were discussed together with the obtained adsorption data, the thicknesses of the guar gum adsorption layer in the presence of surfactants as well as surface charge density and the zeta potential.

Guar gum (GG) is a natural, nonionic, non-toxic polysaccharide, produced from the seeds of the two annual leguminous plants, Cyamopsis tetragonalobus and C. psoraloides (Ma and Pawlik 2007). This polymer contains the units of $\beta$-D-mannopyranose with $\alpha$-D-galactopyranose ones linked in the position $(1 \rightarrow 4)$ and connected to the mannose backbone through $(1 \rightarrow 6)$ glycosidic linkages (Table 1). Polymannose is randomly substituted with galactose. The degree of substitution of polymannose varies from 1.8 to 1.0 (Ma and Pawlik 2005). The average molecular weight of guar gum changes from 1 to 2 million (Cheng et al. 2002). Guar gum is used as a depressant for hydrophobic gangue minerals in flotation of nickel and platinum bearing ores (Wang et al. 2005) or as a blinder of water-insoluble slimes in potash flotation

Table 1 Names and structures of polymers used in the measurements

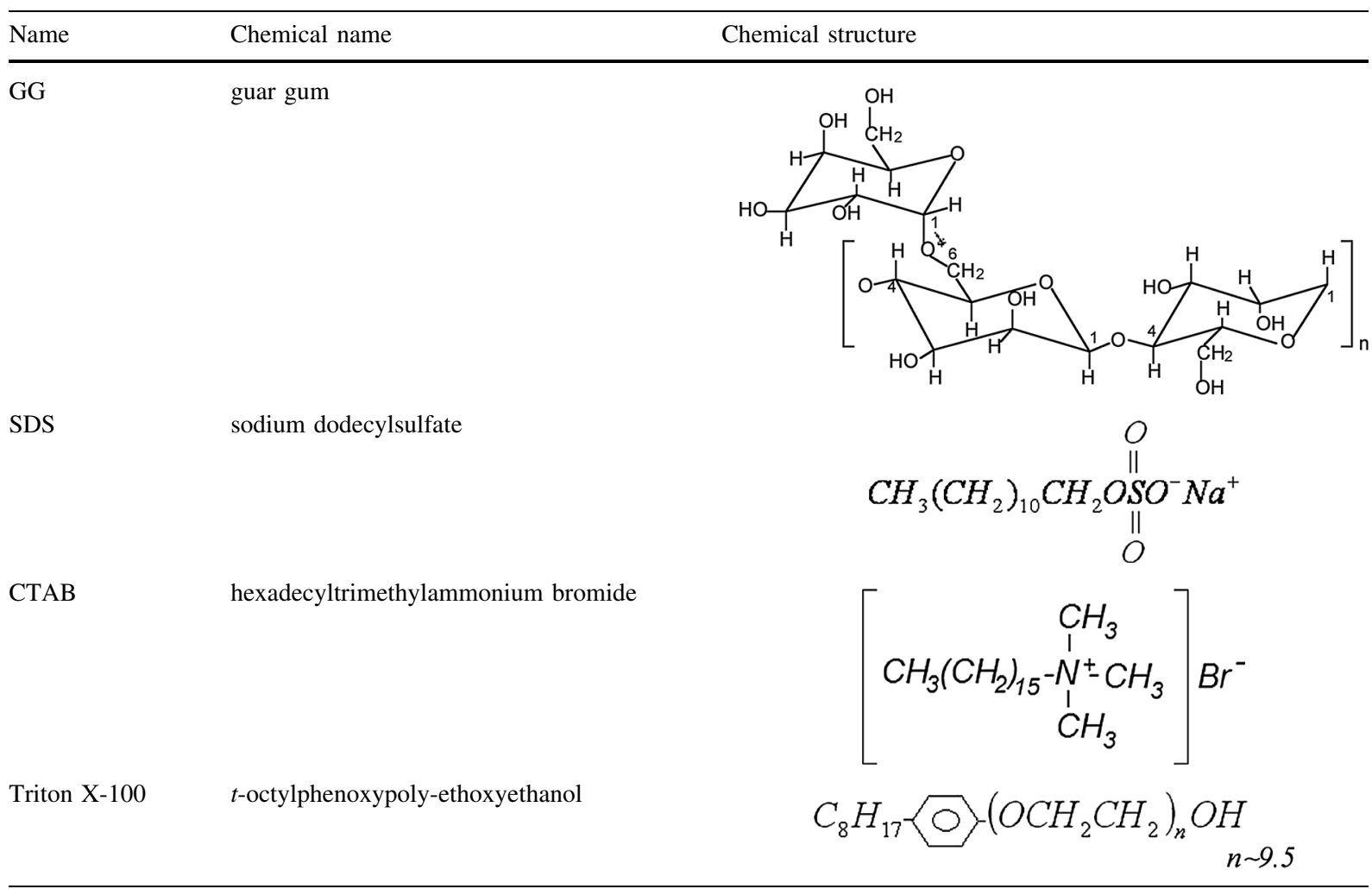


(Wiśniewska et al. 2012a). Manganese dioxide was used as an adsorbent. Its chemical inertness, insolubility in water, stability in a broad $\mathrm{pH}$ range and welldefined interface allow to use this compound as an adsorbent in many adsorption processes. It occurs in nature as a mineral pyrolusite. This oxide finds application in the production of matches, in glassmaking industry for decolourization of glass and as a depolarizer in voltaic cells (Trzebiatowski 1979). Sodium dodecyl sulphate (SDS) $\left(\mathrm{C}_{12} \mathrm{H}_{25} \mathrm{SO}_{4} \mathrm{Na}\right)$ was used as an anionic surfactant. It is applied in industrial products including engine degreasers, car wash soaps as well as in household products such as shampoos, toothpastes and shaving foams (Scheibel 2004). Hexadecyltrimethylammonium bromide (CTAB) was used as a cationic surfactant. Its uses include synthesis of nanoparticles and hair conditioning products (Chakraborty et al. 2006). T-octylphenoxypolyethoxyethanol known as Triton X-100 (TX-100) $\left(\mathrm{C}_{8} \mathrm{H}_{17} \mathrm{C}_{6} \mathrm{H}_{4}\left(\mathrm{OCH}_{2} \mathrm{CH}_{2}\right)_{\mathrm{n}} \times \mathrm{OH} ; \mathrm{n} \sim 9.5\right)$ was used as a nonanionic surfactant in the presented measurements. Triton X-100 is frequently used in stabilization of biochemistry processes (Prete' et al. 2002).

\section{Experimental}

\section{Materials}

$\mathrm{MnO}_{2}$ produced by POCh Gliwice (Poland) was used as an adsorbent. The BET specific surface area for the sample was found to be $35 \mathrm{~m}^{2} \mathrm{~g}^{-1}$. The particle size distribution of the $\mathrm{MnO}_{2}$ sample determined with the use of a Malvern Mastersizer 2000, fell entirely in the range from 1.82 to $22.71 \mu \mathrm{m}$, with a volume average size of $6.78 \mu \mathrm{m}$. The adsorbent was washed with doubly-distilled water until the conductivity of the supernatant was smaller than $2 \mu \mathrm{Scm}^{-1}$. The XRD measurements confirmed that $\mathrm{MnO}_{2}$ was free of impurities.

Guar gum was obtained from Sigma-Aldrich. All guar gum stock solutions were prepared by quickly adding $0.045 \mathrm{~g}$ of gum powder into $45 \mathrm{ml}$ of vigorously stirred water and further stirring for $30 \mathrm{~min}$. The solution was refrigerated overnight to ensure complete hydration or dissolution of guar gum and then filtered through filter paper to remove any undissolved impurities.

SDS, CTAB and Triton X-100 were purchased from Fluka. The concentrations of used surfactants and their mixtures in all measured systems equalled $10^{-4} \mathrm{~mol} \mathrm{dm}^{-3}$. Such a value prevents from exceeding the critical micelle concentration. For SDS the critical micelle concentration equals $0.00825 \mathrm{~mol} \mathrm{dm}^{-3}$ (Rodriguez-Cruz et al. 2005), for CTAB $0.00086 \mathrm{~mol} \mathrm{dm}^{-3}$ (Xi and Guo 2007) whereas for Triton $\mathrm{X}-100-0.00029 \mathrm{~mol} \mathrm{dm}^{-3}$ (Rharbi and Winnik 2001). $\mathrm{NaCl}$ produced by Fluka was used as the supporting electrolyte.

All experiments were carried out in doubly-distilled water at room temperature $\left(\cong 25^{\circ} \mathrm{C}\right)$ because it was proved that the temperature influences the polymer adsorption, electrokinetic properties of the measured system as well as stability of the obtained suspensions (Wiśniewska 2011).

\section{Methods}

\section{Stability measurements}

The measurements of $\mathrm{MnO}_{2}$ suspension stability in the presence and absence of guar gum as well as surfactants and their mixtures were conducted. The $\mathrm{MnO}_{2}$ suspension was prepared by the addition of $0.005 \mathrm{~g}$ of metal oxide to $10 \mathrm{~cm}^{3}$ of a background electrolyte solution $\left(10^{-1} \mathrm{~mol} / \mathrm{dm}^{3} \mathrm{NaCl}\right)$. The obtained suspension was subjected to the action of ultrasounds for $3 \mathrm{~min}$. Then, it was moved to a measuring high glass phial which was placed in a measuring thermostated chamber in the device Turbiscan Lab Expert with the cooling module TLAb Cooler. This apparatus possesses the electroluminescence diode which emits collimated light beam $(\lambda=880 \mathrm{~nm})$ passing through the investigated suspension. It has also two synchronized detectors. The transmission detector recorded light passing through the probe at the angle $0^{\circ}$ in relation to the incident light direction. The other one is the backscattering detector registering the light scattered at the angle $135^{\circ}$. The device is connected with the computer which stores and processes the data. The obtained data are stored and converted by the computer program. The results are presented in the form of curves which show the intensities of transmission and backscattering as a function of time. The analysed suspension in a glass vial (7 cm long) was placed in a thermostated measurement chamber. The $\mathrm{MnO}_{2}$ samples with the polymer or with the polymer and surfactants were prepared in an analogous way. A suitable volume of 
the stock guar gum solution ensuring the final concentrations $10 ; 100$ or $200 \mathrm{ppm}$ was added after suspension ultrasounding. In the case of the $\mathrm{MnO}_{2}$ suspension with guar gum and surfactants besides guar gum (concentration $100 \mathrm{ppm}$ ) a suitable volume of the starting solution of surfactant (of the concentration $10^{-3} \mathrm{~mol} / \mathrm{dm}^{3}$ ) was added ensuring the final concentration $10^{-4} \mathrm{~mol} / \mathrm{dm}^{3}$. Each stability measurement was $15 \mathrm{~h}$ long (single scans were collected every $15 \mathrm{~min}$ ). Because the transmission for all samples was higher than $2 \%$, the stability parameter-the turbiscan stability index (TSI), was calculated from the transmission graphs. This parameter takes into account all single measurements during the experiments and the TSI value is obtained from their averaging. All processes taking place in the sample (including thickness of sediment and clear layer as well as particles settling) were summed up. This parameter was calculated with the special computer program Turbiscan Easy Soft from the following formula:

$T S I=\sqrt{\frac{\sum_{i=1}^{n}\left(x_{i}-x_{B S}\right)^{2}}{n-1}}$

(2) where $x_{i}$-the average backscattering for each minute of measurement, $x_{B S}$ - the average $x_{1}, n$-the number of scans.

The TSI values change in the range from 0 to 100 . The higher the TSI, the more unstable the system is.

\section{Adsorption measurements}

$10 \mathrm{~mL}$ of the solution was prepared from the polymer stock solution (GG), electrolyte $\left(\mathrm{NaCl}\right.$ or $\left.\mathrm{CaCl}_{2}\right)$, doubly-distilled water and surfactants (SDS, CTAB, TX-100 or their mixture with the molar ratio $1: 1$ ). After 15 min (time for complex formation between polysaccharide and surfactant) $0.2 \mathrm{~g}$ of manganese dioxide was added to the solutions. $\mathrm{pH}$ was adjusted to the desired value using $0.1 \mathrm{~mol} \mathrm{dm}^{-3} \mathrm{HCl}$ and 0.1 mol dm ${ }^{-3} \mathrm{NaOH}$. The suspension was shaken for $18 \mathrm{~h}$ to achieve the adsorption-desorption equilibrium by means of a thermostated stirrer. To determine guar gum adsorption amount, the colorimetric method described by Dubois et al. (1956) was used. $0.05 \mathrm{~mL}$ of $80 \%$ phenol and $5 \mathrm{~mL}$ of $98 \%$ sulphuric acid were added to $2 \mathrm{ml}$ of supernatant obtained after centrifugation with the speed 14,000 rpm using a high speed centrifuge (310b Mechanika Precyzyjna). The centrifugation time was $15 \mathrm{~min}$. After $30 \mathrm{~min}$ of colour development, the absorbance was measured at a wavelength of $490 \mathrm{~nm}$ using a spectrophotometer (Cary 100, Varian Instruments). All measurements were made as triplicates. In this paper the average values are reported. The amount of GG adsorption on the $\mathrm{MnO}_{2}$ surface was calculated from a calibration curve according to the concentration difference before and after the adsorption tests.

\section{Thickness of adsorption layer}

The thickness of the polysaccharide adsorption layer $(\delta)$ was determined from the viscosity measurements (M'Pandou and Siffert 1987), using a rheometer (CVO 50 , Bohlin Instruments). Polysaccharide adsorption on the solid surface causes the increase of the solid particle radius which gives the adsorption layer thickness $(\delta)$. It results in the increase of volume fraction $\left(\phi_{0}\right)$ of the dispersed solid. Thus the values $\delta$ were obtained from the dependence:

$\delta=r\left[\left(\frac{\phi_{\mathrm{p}}}{\phi_{0}}\right)^{1 / 3}-1\right]$

where $r$ - the radius of the metal oxide particle, $\phi_{\mathrm{p}}$ the volumetric fraction in the presence of polymer, $\phi_{0}$-the volumetric fraction in the absence of the polymer.

The Einstein equation connects the volume fraction of dispersed solid with the suspension viscosity in the following way:

$\frac{\eta}{\eta_{0}}=1+k \phi_{0}$

where $\eta$-the viscosity of the suspension ( $\mathrm{Pa} \mathrm{s}$ ), $\eta_{0}$ the viscosity of the liquid phase ( $\mathrm{Pa} \mathrm{s}$ ), and $k$-the Einstein coefficient. The coefficient $k$ is equal to 2.5 for the rigid spherical particles in infinitely diluted suspensions.

The volumetric fraction $\left(\phi_{\mathrm{p}}\right)$ in the presence of polymer or polymer-surfactant complex was determined from the linear dependence of $\eta / \eta_{0}$ versus $\phi_{0}$ of manganese dioxide (calibration curve). The viscosity measurements enabling the $\eta / \eta_{0}$ ratio determination in the presence of the polymer or the polymer-surfactant complex were made with the volume fraction of $\mathrm{MnO}_{2}$ equal to $13.7 \times 10^{-3}$. Because the adsorption of 
polymer or polymer surfactant complex caused the increase of the ratio value, $\phi_{\mathrm{p}}$ was determined directly from the calibration curve (as a magnitude related to this ratio). Then the thickness of polysaccharide adsorption layer was calculated (Eq. 2).

\section{Potentiometric titration}

Surface charge on the metal oxide is formed as a result of reactions between the surface hydroxyl groups and the electrolyte ions (Janusz et al. 1997). In aqueous solutions hydrogen/hydroxide ions as well as ions of background electrolyte are the most important in the surface charge formation process. Hydrogen ions influence the surface charge through the acid-base reactions of the surface hydroxyl groups:

$$
\begin{aligned}
& \equiv \mathrm{SOH}_{2}^{+} \longleftrightarrow \equiv \mathrm{SOH}+\mathrm{H}^{+} \\
& \equiv \mathrm{SOH} \longleftrightarrow \equiv \mathrm{SO}^{-}+H^{+}
\end{aligned}
$$

In classic theories of the electrical double layer, the background electrolyte ions are assumed to adsorb non-specifically, but in modern models these ions undergo also the specific adsorption.

Comparison of the titration curves of the same ionic strength is used to determine the surface charge density of metal oxide. The surface charge density is calculated from the dependence between the volume of acid/base added to the suspension in order to obtain the desired $\mathrm{pH}$ value:

$\sigma_{0}=\frac{\Delta V c F}{m S}$

where $\Delta \mathrm{V}$ - the dependence between the volume of acid/base added to the suspension in order to obtain the desired $\mathrm{pH}$ value, $\mathrm{c}$ - the molar concentration of acid/base, F-the Faraday constant $(9.648 \times$ $\left.10^{4} \mathrm{C} \mathrm{mol}^{-1}\right), \mathrm{m}$ - the mass of metal oxide, $\mathrm{S}$ - the specific surface area of metal oxide.

$\mathrm{MnO}_{2}$ surface charge density in the presence and absence of guar gum (10, 100 and $200 \mathrm{ppm})$ and surfactants (CTAB, TX-100, SDS and mixtures CTAB/TX-100 and SDS/TX-100) was determined using the potentiometric titration method. The $\mathrm{NaCl}$ concentration was $0.1 \mathrm{~mol} \mathrm{dm}^{-3}$. A thermostated, Teflon vessel with a shaker, an automatic burette (Dosimat 665, Methrom) and a $\mathrm{pH}$-meter were the parts of the measurement set. The process was controlled by a computer. The density of $\mathrm{MnO}_{2}$ surface charge was determined using the "Miar_t" programme written by W. Janusz. The volume of the measured solution was $50 \mathrm{~cm}^{3}$, the mass of the solid $0.2 \mathrm{~g}$. The surface charge density measurements were made as triplicates for every measured system. The results were obtained with the measurement uncertainty lower than $3 \%$.

\section{Zeta potential measurements}

$0.05 \mathrm{~g}$ of manganese dioxide was added to $500 \mathrm{~cm}^{3}$ of the supporting electrolyte solution $\left(0.1 \mathrm{~mol} \mathrm{dm}^{-3}\right.$ $\mathrm{NaCl})$ with or without $\mathrm{GG}(10,100$ and $200 \mathrm{ppm})$ and the surfactants (CTAB, TX-100, SDS and the CTAB/ TX-100 and SDS/TX-100 mixtures). The obtained suspensions were ultrasonificated for $10 \mathrm{~min}$. Then $\mathrm{pH}$ was adjusted and the electrophoretic mobility was measured using a zetameter (Zetasizer 3,000, Malvern Instruments) and then the zeta potential $(\zeta)$ was calculated from the Smoluchowski equation. The zeta potential measurements were made as triplicates and the results were obtained with the measurement uncertainty from 3 to $7 \%$. In the paper the average values are reported.

\section{Results and discussion}

Stabilization-flocculation properties of the suspension in the presence of polymers and surfactants depend on the conformation assumed by macromolecules and surface active compounds adsorbed on the solid surface. It is the conformation that decides about the polymer adsorption and thickness of its adsorption layer. Figures 1, 2, 3, 4, 5, 6, 7, 8, 9 present the dependence of transmission and backscattering of light after its transition through the manganese dioxide suspensions in the absence of guar gum and surfactants (Fig. 1) and in the presence of this polymer (Figs. 2, 3, 4) as well as in the presence of GG, surfactants or their mixtures (Figs. 5, 6, 7, 8, 9). All these results were obtained using Turbiscan. To estimate the total effect of guar gum and surfactants on the stability of $\mathrm{MnO}_{2}$ suspension, the stability coefficient (TSI-Turbiscan Stability Index) was determined. The calculated values of TSI are given in Table 2. When the system is less stable the value of the stability coefficient is larger. 
Fig. 1 Transmission and backscattering for the system: $\mathrm{MnO}_{2} / 0.1 \mathrm{M} \mathrm{NaCl}$, $\mathrm{pH}=7$
Fig. 2 Transmission and backscattering for the system: $\mathrm{MnO}_{2} / 0.1 \mathrm{M} \mathrm{NaCl} /$ 10 ppm GG, pH = 7

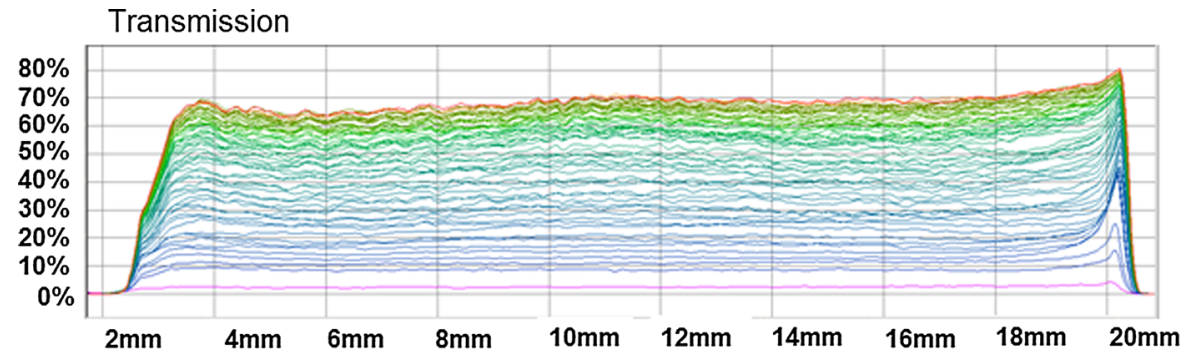

Backscattering

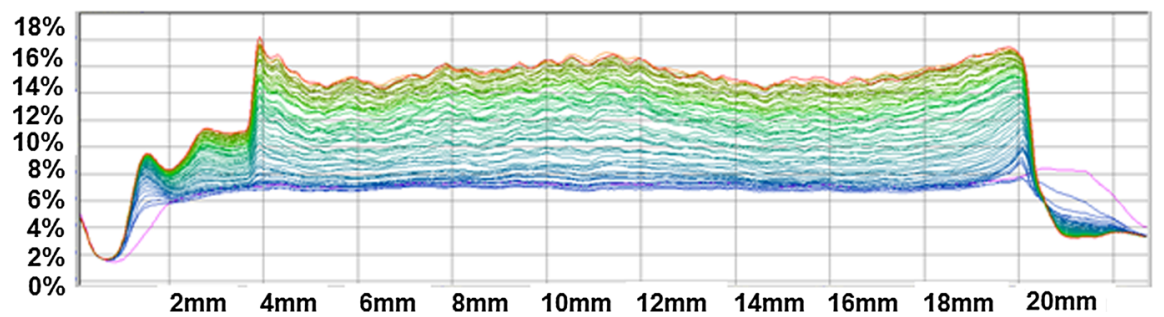

Transmission

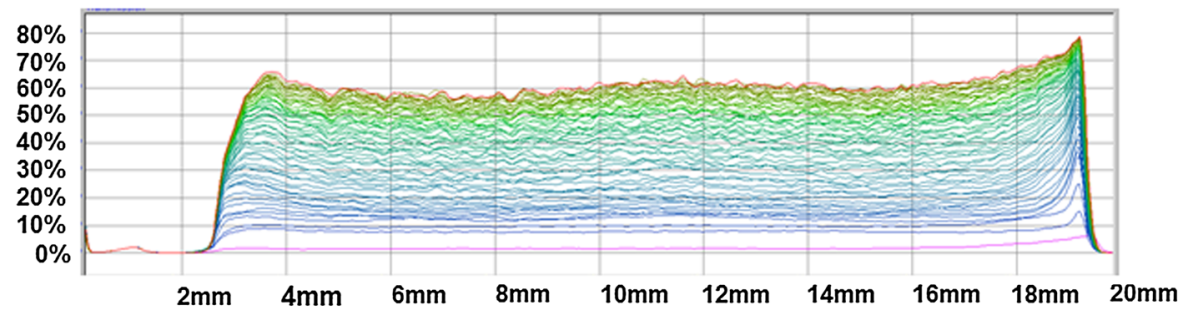

Backscattering

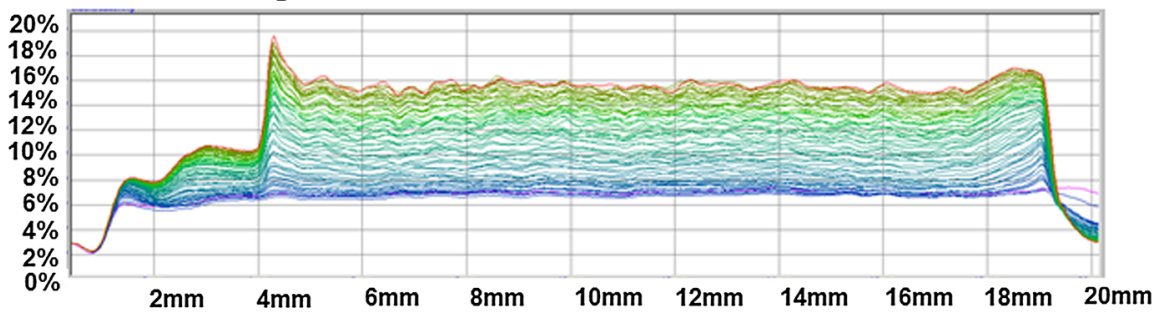

The analysis of the transmission and backscattering curves leads to the conclusion that the $\mathrm{MnO}_{2}$ suspension is quite unstable in the absence of polymer or polymer and surfactants (Fig. 1). The evidence for that is the increase of the transmission of the sample from 2 to $70 \%$ in time of the experiment $(15 \mathrm{~h})$ as well as the high value (40.32) of the TSI parameter for this system (Table 2). The reason for this unstability of the $\mathrm{MnO}_{2}$ suspension is the fact that the absolute values of the surface charge density and the zeta potential (Table 3) are much smaller than in the presence of polymer or polymer and surfactants. Then the repulsive interactions between solid molecules are too weak to provide the stability of the system. Under the measured $\mathrm{pH}(\mathrm{pH}=7)$ manganese dioxide surface is negatively charged, $\mathrm{pH}_{\mathrm{pzc}}$ and $\mathrm{pH}_{\text {iep }}$ points $\approx 4.5-5$ (Grzadka 2010). Because of that the metal oxide particles can repel each other (electrostatic stabilization) but this repulsion is too low to achieve the long-last stability. This type of stabilization is based on the mechanism in which the attractive van der Waals forces are counterbalanced by the repulsive Coulomb forces acting between the negatively charged colloidal particles (Sato and Ruch 1980) 
Fig. 3 Transmission and backscattering for the system: $\mathrm{MnO}_{2} / 0.1 \mathrm{M} \mathrm{NaCl} /$ 100 ppm GG, pH $=7$
Fig. 4 Transmission and backscattering for the system: $\mathrm{MnO}_{2} / 0.1 \mathrm{M} \mathrm{NaCl} /$ 200 ppm GG, pH = 7
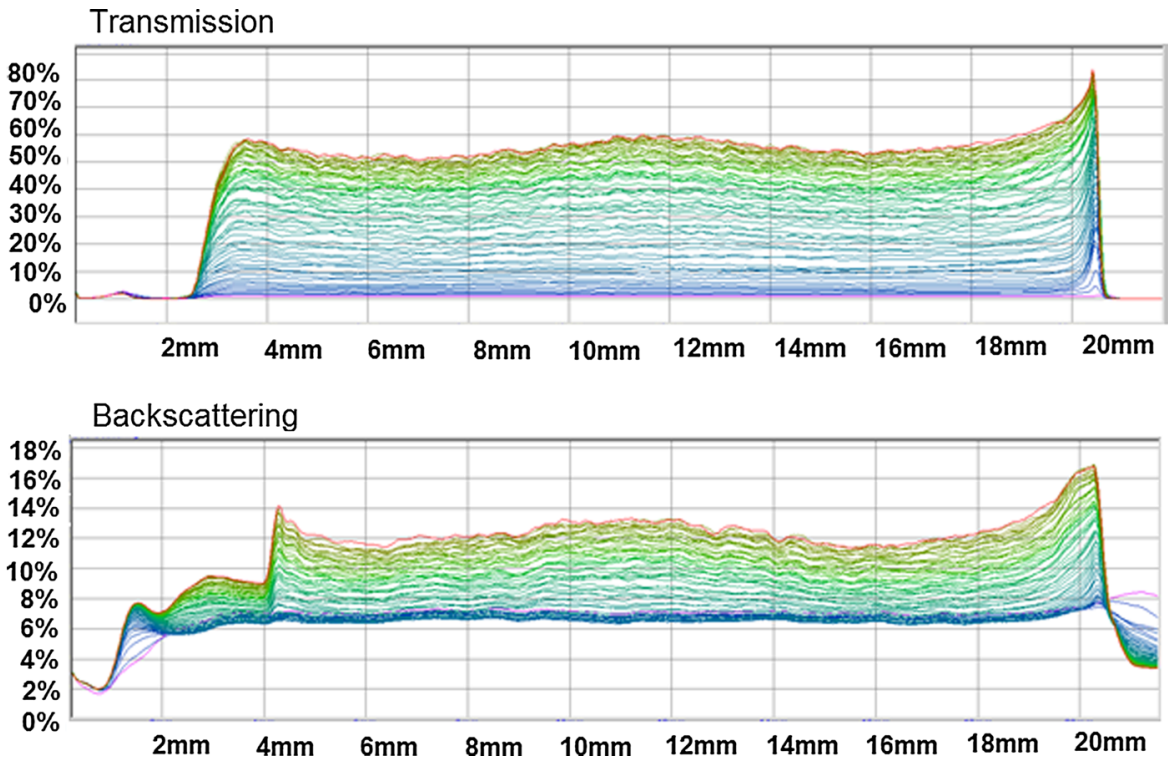

Transmission

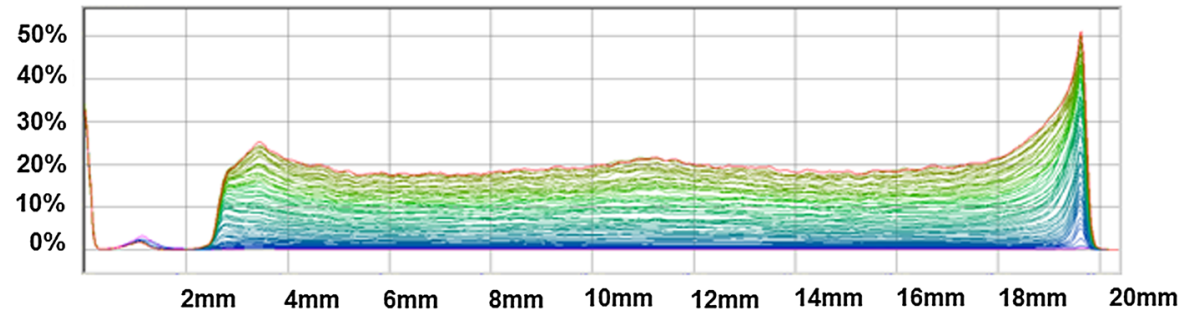

Backscattering

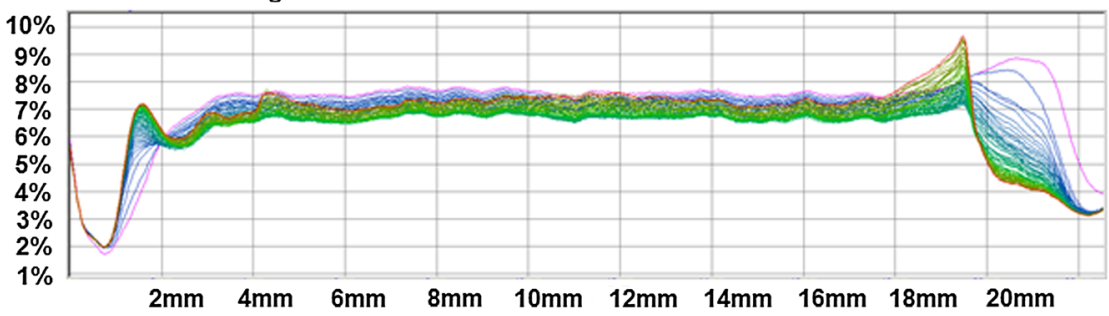

and the particles of metal oxide. They easily fall under the effect of gravity force. The addition of guar gum causes the increase of stability of the $\mathrm{MnO}_{2}$ suspensions. The higher polymer concentration, the more stable the $\mathrm{MnO}_{2}$ suspensions are. The evidence for this fact are lower transmissions (about $60 \%$ for $10 \mathrm{ppm}$ of GG, about $50 \%$ for $100 \mathrm{ppm}$ of GG and only around $20 \%$ for $200 \mathrm{ppm}$ of GG). Moreover, the TSI values are lower than that in the absence of the polymer. They equal $32.59,28.54$ and 8.38 for 10,100 and 200 ppm of GG respectively (Table 2). However, in the case of the system containing $10 \mathrm{ppm}$ of guar gum (Fig. 2), the polymer concentration is too low to provide the stability of the $\mathrm{MnO}_{2}$ suspension. Because of low adsorption of GG as well as low value of the adsorption layer thickness (Table 4) polymer poorly covered the manganese dioxide particles and under such conditions the most possible mechanisms are steric and electrostatic stabilizations sometimes called electrosteric stabilization (Napper 1983). The steric stabilization occurs when the polymer chains are adsorbed on a few solid particles and their adsorption 
Fig. 5 Transmission and backscattering for the system: $\mathrm{MnO}_{2} / 0.1 \mathrm{M} \mathrm{NaCl} /$ $100 \mathrm{ppm} \mathrm{GG/0.0001 \textrm {M }}$ $\mathrm{CTAB}, \mathrm{pH}=7$
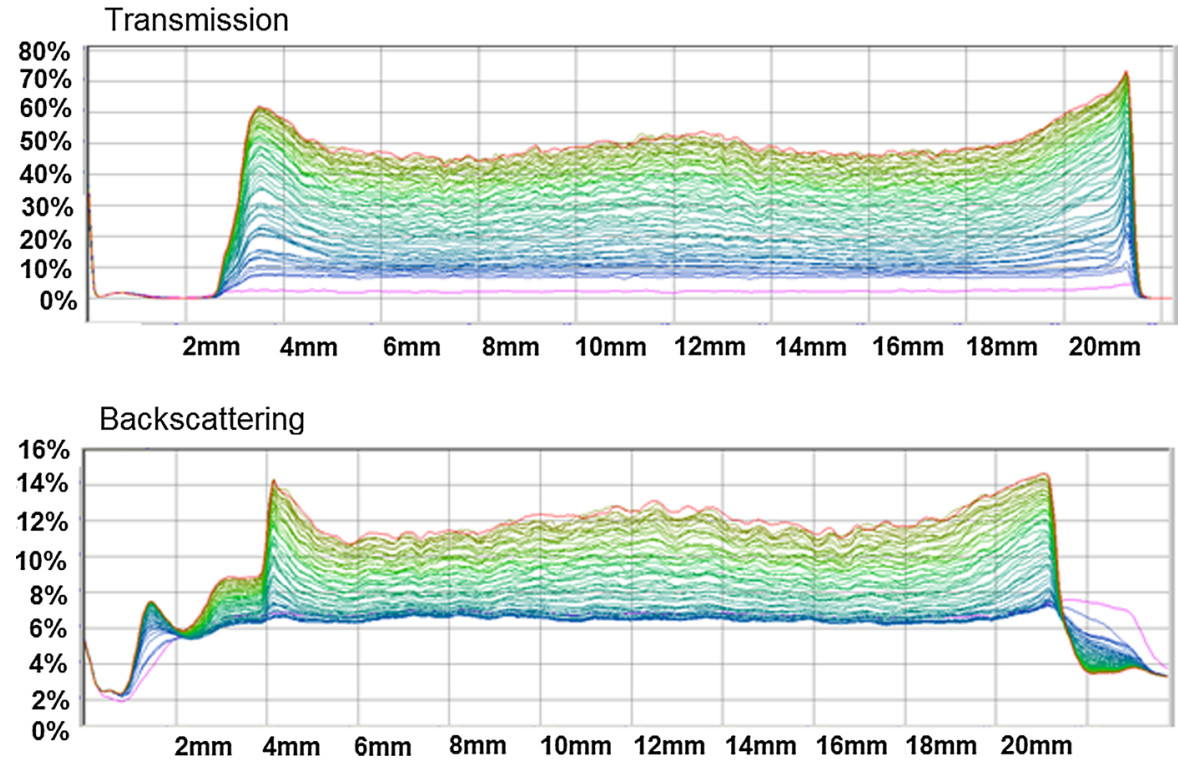

Fig. 6 Transmission and backscattering for the system: $\mathrm{MnO}_{2} / 0.1 \mathrm{M} \mathrm{NaCl} /$ $100 \mathrm{ppm}$ GG/0.0001 M TX$100, \mathrm{pH}=7$
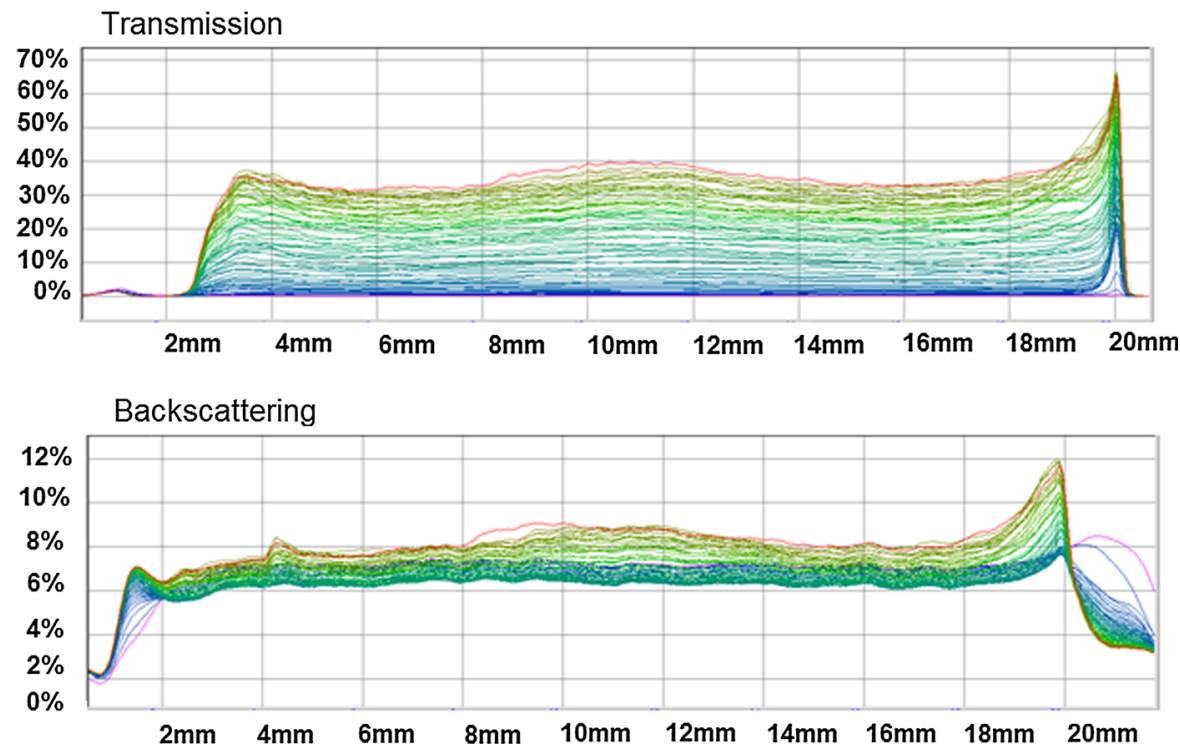

layers can penetrate. This causes the repulsion between the particles, which is the entropic effect. Increasing the concentration of polymer segments in the interpenetrating layers causes the local increase of both the osmotic pressure and the free energy. The consequence of that is the increase in the colloidal stability of the suspensions. In the presence of $100 \mathrm{ppm}$ of guar gum (Fig. 3), the medium stability of the metal oxide suspensions is observed compared to the data obtained for the systems with 10 and $200 \mathrm{ppm}$ of guar gum. These medium results are in agreement with the values of $100 \mathrm{ppm}$ of guar gum adsorption on $\mathrm{MnO}_{2}$ and the thickness created by $100 \mathrm{ppm}$ of GG adsorption layer (Table 4). The highest improvement of the suspension stability occurs in the system containing $200 \mathrm{ppm}$ of GG (Fig. 4). This fact is additionally proved by poor separation of individual scans. In these two cases the most possible mechanism of stabilization is also steric stabilization or combination of steric, electrostatic and depletion stabilization. The last type of stabilization of colloidal suspensions occurs if free polymer 
Fig. 7 Transmission and backscattering for the system: $\mathrm{MnO}_{2} / 0.1 \mathrm{M} \mathrm{NaCl} /$ 100 ppm GG/0.0001 M SDS, $\mathrm{pH}=7$
Fig. 8 Transmission and backscattering for the system: $\mathrm{MnO}_{2} / 0.1 \mathrm{M} \mathrm{NaCl} /$ 100 ppm GG/0.0001 M mixture CTAB/TX-100, $\mathrm{pH}=7$
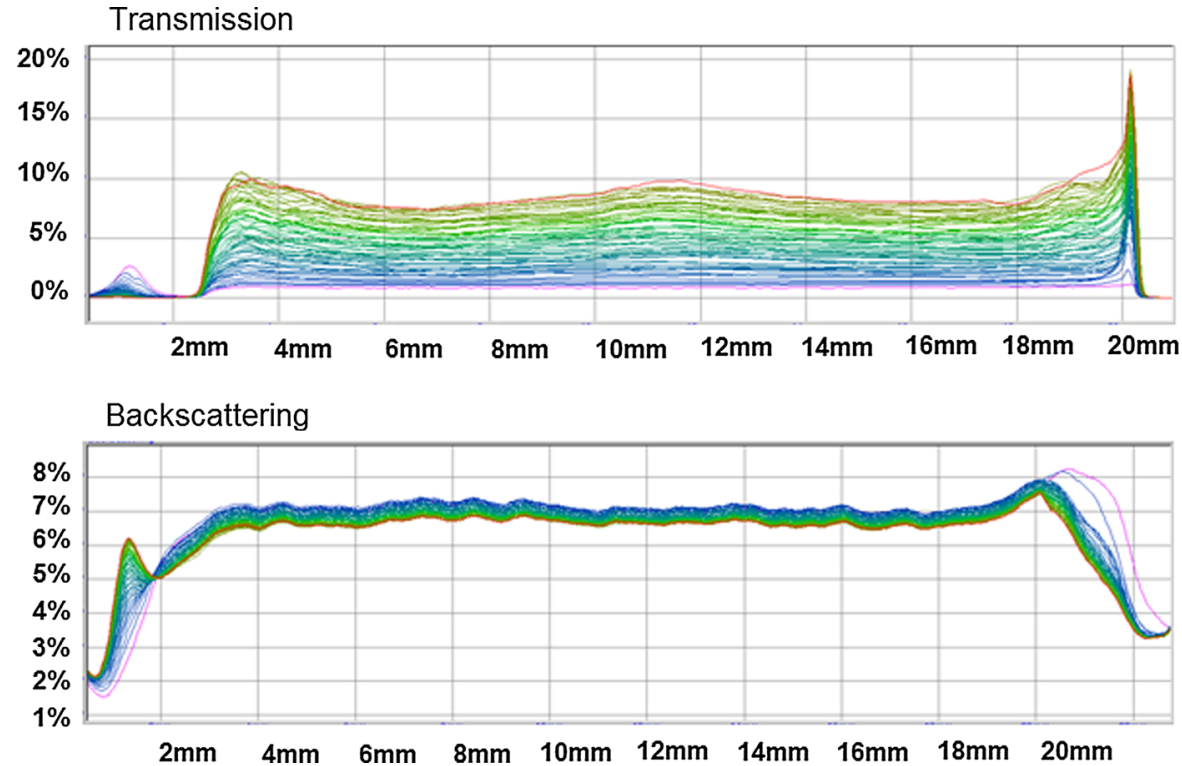

Transmission

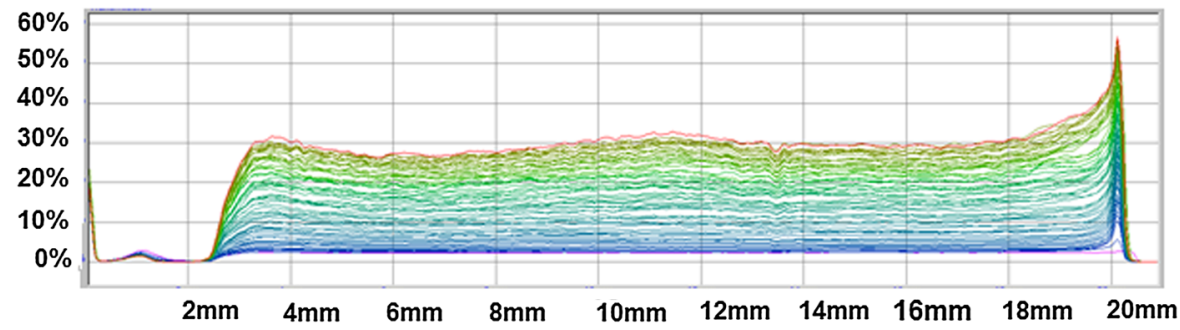

Backscattering

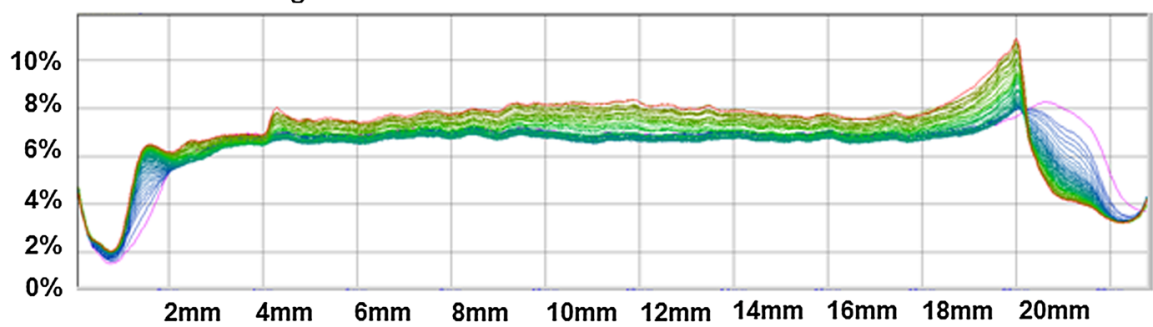

macromolecules which are not adsorbed on $\mathrm{MnO}_{2}$ create repulsive forces between the particles, which prevents the solid particles from colloidal aggregation.

The results presented in Figs. 5, 6, 7 enable the analysis of the influence of single surfactants on the stability of manganese dioxide in the presence of $100 \mathrm{ppm}$ of guar gum. As one can notice, the addition of each free measured surfactant (CTAB, TX-100 and SDS) causes the improvement of stability of the $100 \mathrm{ppm}$ guar gum/ $\mathrm{MnO}_{2}$ system. The TSI values are lower in the systems with the surfactants in comparison to that containing only $100 \mathrm{ppm}$ guar gum. Moreover, the transmission in the systems with TX100 and SDS is lower and equals 30 and $10 \%$ respectively and the transmission in the system with CTAB is at the same level as 100 ppm of GG but with the lower TSI value. The adsorption of polysaccharide in the presence of surfactants increases (Table 4) which is the effect of complex formation between these two adsorbates. The nature of these complexes 
Fig. 9 Transmission and backscattering for the system: $\mathrm{MnO}_{2} / 0.1 \mathrm{M} \mathrm{NaCl} /$ $100 \mathrm{ppm}$ GG/0.0001 M mixture SDS/TX-100, $\mathrm{pH}=7$

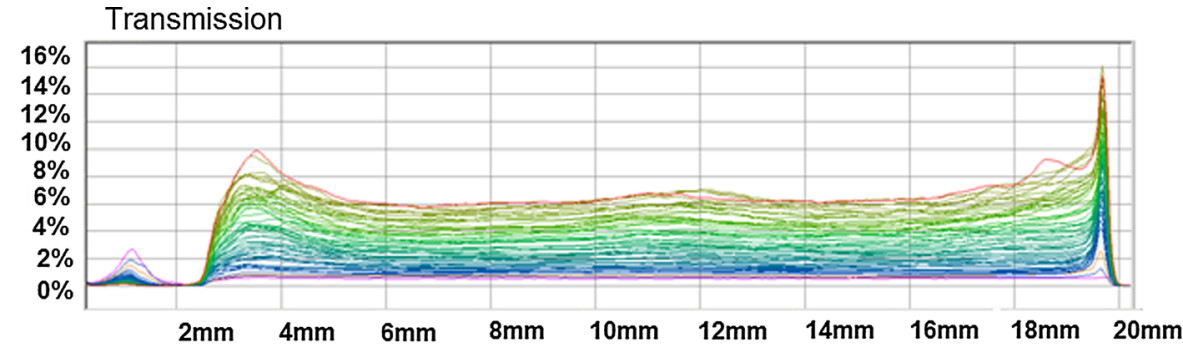

Backscattering

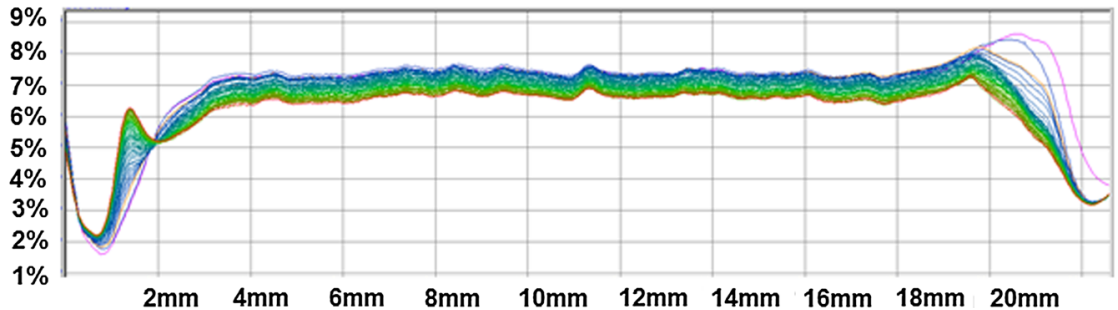

Table 2 Stability coefficient TSI for the $\mathrm{MnO}_{2}$ suspensions in the presence or absence of guar gum and surfactants, $0.1 \mathrm{M}$ $\mathrm{NaCl}, \mathrm{pH}=7$

\begin{tabular}{|c|c|}
\hline System & TSI \\
\hline $\mathrm{MnO}_{2} / 0.1 \mathrm{M} \mathrm{NaCl}$ & 40.32 \\
\hline $\mathrm{MnO}_{2} / 0.1 \mathrm{M} \mathrm{NaCl} / 10 \mathrm{ppm}$ GG & 32.59 \\
\hline $\mathrm{MnO}_{2} / 0.1 \mathrm{M} \mathrm{NaCl} / 100 \mathrm{ppm} \mathrm{GG}$ & 28.54 \\
\hline $\mathrm{MnO}_{2} / 0.1 \mathrm{M} \mathrm{NaCl} / 200 \mathrm{ppm}$ GG & 8.38 \\
\hline $\mathrm{MnO}_{2} / 0.1 \mathrm{M} \mathrm{NaCl} / 100$ ppm GG/0.0001 M CTAB & 23.71 \\
\hline $\mathrm{MnO}_{2} / 0.1 \mathrm{M} \mathrm{NaCl} / 100$ ppm GG/0.0001 M TX-100 & 15.09 \\
\hline $\mathrm{MnO}_{2} / 0.1 \mathrm{M} \mathrm{NaCl} / 100 \mathrm{ppm}$ GG/0.0001 M SDS & 3.96 \\
\hline $\begin{array}{l}\mathrm{MnO}_{2} / 0.1 \mathrm{M} \mathrm{NaCl} / 100 \mathrm{ppm} \text { GG/0.0001 M mixture } \\
\mathrm{CTAB} / \mathrm{TX}-100\end{array}$ & 12.84 \\
\hline $\begin{array}{l}\mathrm{MnO}_{2} / 0.1 \mathrm{M} \mathrm{NaCl} / 100 \mathrm{ppm} \text { GG/0.0001 M mixture } \\
\mathrm{SDS} / \mathrm{TX}-100\end{array}$ & 2.89 \\
\hline
\end{tabular}

depends on the chemical character of both the polymer and the surfactants and was discussed in my earlier papers (Grządka and Chibowski 2012; Grzadka 2013). What is more, the created adsorption layer in the presence of surfactants is more expanded towards the liquid phase than in the case of the system containing only $100 \mathrm{ppm}$ GG. This observation might be confirmed by the obtained data of the adsorption layer thickness (Table 4). It is also known that the structure of the created complexes is as follows: GG-surfactantGG-surfactant which means that polysaccharide is directly bonded to the surface of the solid and surfactants molecules are present in the upper parts
Table 3 Surface charge density $(\sigma)$ and the zeta potential $(\zeta)$ of manganese dioxide in the presence and absence of guar gum and surfactants, $0.1 \mathrm{M} \mathrm{NaCl}, \mathrm{pH}=7$

\begin{tabular}{lcc}
\hline System & $\begin{array}{l}\text { Surface } \\
\text { charge } \\
\text { density } \\
\left(\mu \mathrm{C} / \mathrm{cm}^{2}\right)\end{array}$ & $\begin{array}{l}\text { Zeta } \\
\text { potential } \\
(\mathrm{mV})\end{array}$ \\
\hline $\mathrm{MnO}_{2} / 0.1 \mathrm{M} \mathrm{NaCl}$ & -18.33 & -3.17 \\
$\mathrm{MnO}_{2} / 0.1 \mathrm{M} \mathrm{NaCl} / 10 \mathrm{ppm} \mathrm{GG}$ & -18.39 & -14.78 \\
$\mathrm{MnO}_{2} / 0.1 \mathrm{M} \mathrm{NaCl} / 100 \mathrm{ppm} \mathrm{GG}$ & -18.42 & -24.3 \\
$\mathrm{MnO}_{2} / 0.1 \mathrm{M} \mathrm{NaCl} / 200 \mathrm{ppm} \mathrm{GG}$ & -18.41 & -32.2 \\
$\mathrm{MnO}_{2} / 0.1 \mathrm{M} \mathrm{NaCl} / 100 \mathrm{ppm} \mathrm{GG} /$ & -18.36 & 5.27 \\
$0.0001 \mathrm{M} \mathrm{CTAB}$ & & \\
$\mathrm{MnO}_{2} / 0.1 \mathrm{M} \mathrm{NaCl} / 100 \mathrm{ppm} \mathrm{GG} /$ & -18.54 & -25.9 \\
$0.0001 \mathrm{M} \mathrm{TX}-100$ & & -29.4 \\
$\mathrm{MnO}_{2} / 0.1 \mathrm{M} \mathrm{NaCl} / 100 \mathrm{ppm} \mathrm{GG} /$ & -18.41 & \\
$0.0001 \mathrm{M} \mathrm{SDS}$ & & 4.36 \\
$\mathrm{MnO}_{2} / 0.1 \mathrm{M} \mathrm{NaCl} / 100 \mathrm{ppm} \mathrm{GG} /$ & -18.45 & \\
$0.0001 \mathrm{M} \mathrm{mixture} \mathrm{CTAB} / \mathrm{TX}-100$ & & -32.6 \\
$\mathrm{MnO}_{2} / 0.1 \mathrm{M} \mathrm{NaCl} / 100 \mathrm{ppm} \mathrm{GG} /$ & -18.33 & \\
$0.0001 \mathrm{M}$ mixture SDS/TX-100 & & \\
\hline
\end{tabular}

of the electrical double layer (Grzadka 2013). Such a conclusion might be drawn on the basis of the elektrokinetic results (Table 3). As one can see the surface charge density of $\mathrm{MnO}_{2}$ is more or less the same in the presence of GG and surfactants of different chemical character but the zeta potential (diffused part of the electric double layer) changes dramatically in the presence of different surfactants. This is the 
Table 4 Adsorbed amounts and thicknesses of guar gum adsorption layers on the $\mathrm{MnO}_{2}$ surface in the presence or absence of surfactants, $0.1 \mathrm{M} \mathrm{NaCl}, \mathrm{pH}=7$

\begin{tabular}{llc}
\hline System & $\Gamma\left(\mathrm{mg} / \mathrm{m}^{2}\right)$ & $\delta(\mathrm{nm})$ \\
\hline $\mathrm{MnO}_{2} / 0.1 \mathrm{M} \mathrm{NaCl} / 10 \mathrm{ppm} \mathrm{GG}$ & 0.002 & 2.3 \\
$\mathrm{MnO}_{2} / 0.1 \mathrm{M} \mathrm{NaCl} / 100 \mathrm{ppm} \mathrm{GG}$ & 0.014 & 6.9 \\
$\mathrm{MnO}_{2} / 0.1 \mathrm{M} \mathrm{NaCl} / 200 \mathrm{ppm} \mathrm{GG}$ & 0.037 & 20.1 \\
$\mathrm{MnO}_{2} / 0.1 \mathrm{M} \mathrm{NaCl} / 100 \mathrm{ppm} \mathrm{GG} /$ & 0.057 & 11.5 \\
$\quad 0.0001 \mathrm{M} \mathrm{CTAB}$ & & \\
$\mathrm{MnO}_{2} / 0.1 \mathrm{M} \mathrm{NaCl} / 100 \mathrm{ppm} \mathrm{GG} /$ & 0.023 & 13.9 \\
$0.0001 \mathrm{M} \mathrm{TX}-100$ & & \\
$\mathrm{MnO}_{2} / 0.1 \mathrm{M} \mathrm{NaCl} / 100 \mathrm{ppm} \mathrm{GG} /$ & 0.051 & 16.2 \\
$0.0001 \mathrm{M} \mathrm{SDS}$ & & \\
$\mathrm{MnO}_{2} / 0.1 \mathrm{M} \mathrm{NaCl} / 100 \mathrm{ppm} \mathrm{GG} /$ & 0.059 & 19.5 \\
$0.0001 \mathrm{M}$ mixture CTAB/TX-100 & & \\
$\mathrm{MnO}_{2} / 0.1 \mathrm{M} \mathrm{NaCl} / 100 \mathrm{ppm} \mathrm{GG} /$ & 0.062 & 20.3 \\
$0.0001 \mathrm{M}$ mixture SDS/TX-100 & & \\
\hline
\end{tabular}

evidence that guar gum is directly connected with the surface of the metal oxide and surfactants (linked to polysaccharide) are present in the diffused part of the electrical double layer. From the comparison of the results of the $\mathrm{MnO}_{2}$ suspensions stability, it is visible that in the presence of cationic CTAB the increase of the stability of the $\mathrm{MnO}_{2} / 100$ ppm GG system is the smallest among single surfactants, in the presence of nonionic TX-100 its value is medium and it is the highest when anionic SDS is added to the adsorption system. The values of the TSI parameter equal 23.71 in the presence of CTAB, 15.09 in the presence of TX-100 and 3.96 after the addition of SDS to the suspension. As follows the anionic surface active agent has the best effectiveness in the stabilizing polymer/metal oxide suspension, cationic surfactant is less effective whereas nonionic one presents the average properties. The reason for this phenomenon is connected with the amount of adsorption of polysaccharide in the presence of these surfactants and with the conformation of the created adsorption layer. Cationic CTAB added to the system easily forms complexes with nonionic GG (adsorption of GG in the presence of CTAB is high) but the adsorption of such a complex on the surface of manganese dioxide causes that the created adsorption layer is compact and thin because of the electrostatic attraction between CTAB and $\mathrm{MnO}_{2}$ which is confirmed by small values of the adsorption layer thickness in the $\mathrm{MnO}_{2} / 0.1 \mathrm{M}$ $\mathrm{NaCl} / 100 \mathrm{ppm}$ GG/0.0001 M CTAB system (Table 4). For of the above mentioned reasons, CTAB is worse than TX-100 or SDS for the improvement of $\mathrm{MnO}_{2}$ stability. In the case of nonionic TX-100 the influence of this surface active agent on the GG adsorption is rather small but the formed adsorption layer is more expanded towards the liquid phase (high values of the adsorption layer thickness) (Table 4). Under such conditions the $\mathrm{MnO}_{2} / 100$ ppm GG system is more stable. However, as mentioned earlier, the anionic surfactant provides the highest stability of the suspension. In this case the adsorption of GG is almost as high as in the presence of CTAB but because of electrostatic repulsion between the negatively charged surfactant and the surface of $\mathrm{MnO}_{2}$, the thickness of the adsorption layer is high. Such a conformation of the adsorption layer expanded and containing a lot of polymer ensures the best stability of the system.

Taking into account the influence of the surfactant mixtures on the stability of $\mathrm{MnO}_{2} / 100 \mathrm{ppm} \mathrm{GG}$ suspensions one might notice that the addition of the surface active agents mixture to the suspension causes the increase of stability not only compared to the system with $100 \mathrm{ppm}$ of GG but also to those containing single surfactants. The TSI values equal 12.84 and 2.89 for the CTAB/TX-100 and SDS/TX100 systems respectively. The transmission in the case of the system with the mixture of cationic and nonionic surfactant reaches $30 \%$ whereas in the latter system only $6 \%$. The explanation of this fact is connected with the synergistic effect of the surfactant mixtures on the adsorption of guar gum. It is known that the synergetic effect involves the increase of adsorptive, foaming and rewetting properties of surfactant mixtures in comparison to pure surfactant solutions (Wang and Kwak 1999; Reif and Somasundaran 1999; Soriyan et al. 2009). As mentioned earlier, the interactions between TX-100 and GG are small, but the presence of another ionic surfactant causes the increase of these interactions. The reason for that is the fact that the electrostatic interactions of ionic surfactants provide a sufficient number of hydrophobic sites for adsorption of nonionic surfactant (Somasundaran and Huang 1997). The obtained results are in agreement with the findings of Palla and Shah (2002) who noticed that mixtures of ionic and non-ionic surfactants are capable of stabilizing suspensions whereas the same surfactants added singlely to the suspensions are not so effective. Among two studied surfactant mixtures better improvement of stabilization was 
obtained in the presence of SDS/TX-100 mixture. Adsorption of guar gam in the presence of this mixture and the thickness of the created adsorption layer are the highest among all measured systems (Table 4). As a result, stability of the measured suspension is the highest too. In the case of CTAB/TX-100 the synergistic effect is visible but stability of the $\mathrm{MnO}_{2}$ suspension is lower in comparison to the previously discussed system. The reason for that is lower adsorption of guar gum in the presence of the CTAB/TX-100 mixture and electrostatic attraction between the polymer-surfactant complex and the surface of the adsorbent which causes that the adsorption layer is thinner.

\section{Conclusions}

The addition of guar gum (in the concentration range 10-200 ppm) to the $\mathrm{MnO}_{2}$ suspensions increases their stability. The highest increase is observed in the presence of $200 \mathrm{ppm}$ of GG. The reason for that is high adsorption and good coverage of metal oxide particles by polymer. However, the mixtures of guar gum and surfactants (CTAB, TX-100, SDS) are even better in stabilizing the above-mentioned suspension. Explanation of this phenomenon might be found in the increase of guar gum adsorption in the presence of surfactants, which results from multilayer complex formation. Among the single surfactants, the most effective one was SDS. It was the best in improving stability of the $\mathrm{MnO}_{2} / 100 \mathrm{ppm}$ GG suspension. The adsorption of guar gum in the presence of this anionic surface active agent is high and the obtained adsorption layer is thick, which provides good stability. The most stable suspensions of $\mathrm{MnO}_{2} / 100 \mathrm{ppm}$ GG are obtained in the presence of surfactant mixtures. The reason for that is the synergetic effect between the mixtures of cationic- nonionic surfactants as well as between the anionic and nonionic ones.

Acknowledgments This research was supported by the Polish Ministry of Science and Higher Education, Project Number: N N204 090438.

Open Access This article is distributed under the terms of the Creative Commons Attribution License which permits any use, distribution, and reproduction in any medium, provided the original author(s) and the source are credited.

\section{References}

Besra L, Sengupta DK, Roy SK, Ay P (2002) Studies on flocculation and dewatering of kaolin suspensions by anionic polyacrylamide flocculant in the presence of some surfactants. Int J Miner Process 66:1-28

Chakraborty I, Malik PK, Moulik SP (2006) Preparation and characterization of $\mathrm{CoS}_{2}$ nanomaterial in aqueous cationic surfactant medium of cetyltrimethylammonium bromide (CTAB). J Nanopart Res 8:889-897

Cheng Y, Brown KM, Prud'homme RK (2002) Characterization and intermolecular interactions of hydroxypropyl guar solutions. Biomacromolecules 3:456-461

Derajaguin BV, Landau LD (1941) Theory of the stability of strongly charged lyophobic sols and of the adhesion of strongly charged particles in solution of electrolytes. Acta Physicochim 14:633-662

Dubois M, Gilles A, Hamilton JK, Rebers PA, Smith F (1956) Colorimetric method for determination of sugar and related substances. Anal Chem 28:350-356

Esumi K, litaka M, Torigoe K (2000) Kinetics of simultaneous adsorption of poly(vinylpyrrolidone) and sodium dodecyl sulfate on alumina particles. J Colloid Interface Sci 232:71-75

Fan A, Somasundaran P, Turro NJ (1999) Role of sequential adsorption of polymer/surfactant mixtures and their mixtures and their conformation in dispersion/flocculation of alumina. Colloids Surf 146:397-403

Grzadka E (2010) The adsorption layer in the system: carboxymethylcellulose/surfactants/ $\mathrm{NaCl} / \mathrm{MnO}_{2}$. J Surfactants Deterg 15:513-521

Grządka E (2011) Competitive adsorption in the system: carboxymethylcellulose/surfactant/electrolyte/ $\mathrm{Al}_{2} \mathrm{O}_{3}$. Cellulose 18:291-308

Grzadka E (2013) Influence of surfactants on the adsorption and elektrokinetic properties of the system: guar gum/manganese dioxide. Cellulose 20:1313-1328

Grządka E, Chibowski S (2012) Adsorption and electrokinetic properties of the system: carboxymethylcellulose/manganese oxide/surfactant. Cellulose 19:23-36

Janusz W, Kobal I, Sworska A, Szczypa J (1997) Investigation of electrical double layer in a metal oxide/monovalent electrolyte solution. J Colloids Interface Sci 187:381-387

M'Pandou A, Siffert B (1987) Polyethyleneglycol adsorption at the $\mathrm{TiO}_{2}-\mathrm{H}_{2} \mathrm{O}$ interface: distortion of ionic structure and shear plane position. Colloids Surf 24:159-172

Ma X, Pawlik M (2005) Effect of alkali metal cations on adsorption of guar gum onto quartz. J Coll Interface Sci 289:48-55

Ma X, Pawlik M (2007) Role of background ions in guar gum adsorption on oxide minerals and kaolinite. J Coll Interface Sci 313:440-448

Napper DH (1983) Polymeric stabilization of colloidal dispersion. Academic Press, New York

Ouali L, Francois J, Pefferkorn E (1999) Adsorption of telechelic Poly(ethylene oxide) on colloids: influence on colloid stability. J Colloid Interface Sci 215:36-42

Palla B, Shah DO (2002) Stabilization of high ionic strength slurries using surfactant mixtures: molecular factors that determine optimal stability. J Colloid Interface Sci 256:143-152 
Prete' $^{\prime}$ PSC, Malheiros SVP, Meirelles NC, de Paula E (2002) Quantitative assessment of human erythrocyte membrane solubilization by Triton X-100. Biophys Chem 97:1-5

Reif I, Somasundaran P (1999) Asymmetric excess free energies and variable interaction parameters in mixed micellization. Langmuir 15:3411-3417

Rharbi Y, Winnik MA (2001) Solute exchange between surfactant micelles by micelle fragmentation and fusion. Adv Colloid Interface Sci 89-90:25-46

Rodriguez-Cruz MS, Sanchez-Martin MJ, Sanchez-Camazano M (2005) A comparative study of adsorption of an anionic and a non-ionic surfactant by soils based on physicochemical and mineralogical properties of soils. Chemosphere 61:56-64

Şakar-Deliormanlı A (2007) Synergistic effect of polymer-surfactant mixtures on the stability of aqueous silica suspensions. J Eur Ceram Soc 27:611-618

Sato T, Ruch R (1980) Stabilization of colloidal dispersion by polymer adsorption. Marcel Dekker Inc., New York

Scheibel JJ (2004) The evolution of anionic surfactant technology to meet the requirements of the laundry detergent industry. J Surfactants Deterg 7:319-328

Somasundaran P, Huang L (1997) Adsorption behavior of surfactant mixtures at solid-liquid interface. Pol $\mathrm{J}$ Chem 71:568-582

Somasundaran P, Yu X, Krishnakumar S (1998) Role of conformation and orientation of surfactants and polymers in controlling flocculation and dispersion of aqueous and nonaqueous suspensions. Colloids Surf 133:125-133

Soriyan OO, Owoyomi O, Bamgbose JT (2009) The effect of mixed surfactants of sodium dodecyl sulphate and TX-100 on the base hydrolysis of Malachite green. React Kinet Catal Lett 98:77-82
Terayama H, Okumura K, Sakai K, Torigoe K, Esumi K (2001) Aqueous dispersion behavior of drug particles by addition of surfactant and polymer. Colloids Surf 20:73-77

Trzebiatowski W (1979) Chemia nieorganiczna PWN, Warszawa

Verwey EJW, Overbeek JThG (1948) Theory of stability of lyophobic colloids. Elsevier, Amsterdam

Wang W, Kwak JCK (1999) Adsorption at the alumina-water interface from mixed surfactant solutions. Colloids Surf 156:95-110

Wang J, Somasundaran P, Nagaraj DR (2005) Adsorption mechanism of guar gum at solid-liquid interfaces. Miner Eng 18:77-81

Wiśniewska M (2011) The temperature effect on electrokinetic properties of the silica-polyvinyl alcohol (PVA) system. Colloid Polym Sci 289:341-344

Wiśniewska M, Chibowski S, Urban T (2010) Influence of solution $\mathrm{pH}$ on stability of aluminium oxide suspension in the presence of polyacrilic acid. Adsorption 16:321-332

Wiśniewska M, Chibowski S, Urban T (2012a) Investigation of the stability of an alumina suspension in the presence of ionic polyacrylamide. Thin Solid Films 520:6158-6164

Wiśniewska M, Chibowski S, Urban T (2012b) Effect of the type of polymer functional groups on the structure of its film formed on the alumina surface—suspension stability. React Funct Polym 72:791-798

Wiśniewska M, Terpiłowski K, Chibowski S, Urban T, Zarko V, Gun'ko VM (2013) Effect of solution pH on the stability of mixed silica- alumina suspension in the presence of polyacrylic acid (PAA) with different molecular weights. Cent Eur J Chem 11:101-110

Xi J, Guo R (2007) Acid-base equilibrium of puerarin in CTAB micelles. J Pharm Biomed Anal 43:111-118 